\title{
Progressões aritméticas de ordem superior
}

\author{
José Nobre Rogério Rocha
}

\section{Resumo}

Este artigo apresenta um estudo das Progressões Aritméticas (PAs) de ordem superior. Mais especificamente, mostraremos que a fórmula do termo geral dessas PAs permite, de forma simples, a resolução de diversos problemas interessantes que aparecem na literatura, a exemplo daqueles que apresentaremos como aplicações. Devido à carência de material que aborda esse conteúdo de forma satisfatória, este artigo servirá de apoio para os professores de Matemática do Ensino Médio.

Palavras-chave: Progressões Aritméticas. Ordem Superior. Termo Geral. Aplicações.

\section{Abstract}

This paper presents a study of Arithmetic Progressions (APs) of higher order. More specifically, we will show that the general term formula of these APs allows, in a simple way, the resolution of several interesting problems that appear in the literature, such as those that we present as applications. Due to the shortage of material that addresses this content in a satisfactory way, this article will be of support to teachers of Mathematics in High School.

Keywords: Arithmetic Progressions. Higher order. General term. Applications

\section{Introdução}

A história mostra que as Progressões Aritméticas (PAs) fazem parte da trajetória humana desde o surgimento da contagem, pois o próprio conjunto dos números naturais representa uma PA.

As sequências dos Números Figurados podem ter sido as primeiras manifestações de PAs de segunda ordem [6]. Os Números Figurados são números que podem ser representados por uma construção geométrica de pontos equidistantes. Se essa representação forma um polígono regular, esses números chamam-se Números Poligonais, dentre os quais destacamos os números triangulares, quadrados, pentagonais, hexagonais etc. A Figura 1 mostra um desenho medieval retratando os Números Poligonais. Mais detalhes históricos podem ser encontrados em [5, Seção 2] e [6].

Dos trabalhos existentes na literatura, relacionados a PAs de ordem superior, destacamos os seguintes:

- [2]: apresenta diversos resultados teóricos e ilustrações relacionados às PAs de ordem superior.

- [3]: desde que todo termo geral de uma PA de ordem $k$ pode ser identificado com um polinômio 


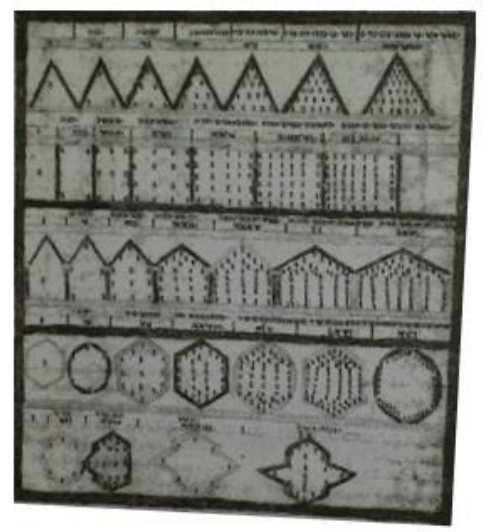

Figura 1: Manuscrito medieval contendo Números Figurados, FONTE: [1].

de grau $k$ [3, Prop. 1], Lima propôs um estudo das PAs de ordem superior no qual os problemas propostos são resolvidos considerando o termo geral como um polinômio de grau $k$. Nesse sentido, os problemas são modelados como sistemas de $(k+1) \times(k+1)$ equações lineares.

- [5]: nesse trabalho, o autor propõe uma abordagem em que os problemas propostos envolvendo PAs de ordem superior são resolvidos utilizando uma fórmula explícita para o termo geral que envolve coeficientes binomiais [5, Prop. 3.11].

Os trabalhos [3] e [5] são dissertações do Programa de Mestrado Profissional em Matemática (PROFMAT). Esses trabalhos utilizam abordagens distintas, que se complementam. Além disso, quando se resolve os problemas pela abordagem utilizada em [5], a solução torna-se mais simples (esse fato pode ser conferido no Exemplo 2 e na Observação 1, nos quais o termo geral de uma PA de ordem 4 é calculado de acordo com as duas abordagens).

Seguindo a abordagem de [5], vamos propor, neste trabalho, um estudo das PAs de ordem superior, apresentando a teoria básica necessária e diversas situações problema, que serão solucionadas com o auxílio da fórmula do termo geral que envolve coeficientes binomiais.

De forma geral, os livros didáticos de Matemática existentes na literatura nacional não abordam o tema "Progressões Aritméticas de ordem superior". Nesse sentido, este trabalho, em conjunto com os demais citados acima, servirão de apoio para os professores do Ensino Médio.

\subsection{Aspectos teóricos}

Nesta seção, apresentaremos os conceitos e resultados que serão fundamentais para o desenvolvimento do nosso trabalho. Mais detalhes podem ser conferidos, por exemplo, em [2], [3], [4] e [5]. 
Definição 1 (Operador Diferença). Dada uma sequência $\left(a_{n}\right)_{n \in \mathbb{N}}$, define-se o chamado operador diferença $\left(\Delta^{1} a_{n}\right)_{n \in \mathbb{N}}=\left(a_{n+1}-a_{n}\right)_{n \in \mathbb{N}}$, que constitui uma nova sequência. Como $\left(\Delta^{1} a_{n}\right)_{n \in \mathbb{N}}$ forma uma nova sequência, podemos novamente obter o operador diferença, isto é, $\left(\Delta^{1}\left[\Delta^{1} a_{n}\right]\right)_{n \in \mathbb{N}}$ $=\left(\Delta^{2} a_{n}\right)_{n \in \mathbb{N}}$, e assim recursivamente, $\left(\Delta^{k} a_{n}\right)_{n \in \mathbb{N}}$, para $k \geq 3$.

Definição 2 (Ordem de uma PA). Uma sequência $\left(a_{n}\right)_{n \in \mathbb{N}}$ será uma PA de ordem $k$ se for necessário aplicar o operador diferença $k$ vezes para se chegar a uma sequência constante.

Desse modo, uma sequência $\left(a_{n}\right)_{n \in \mathbb{N}}$ será uma PA de ordem 1 se o operador diferença $\left(\Delta^{1} a_{n}\right)_{n \in \mathbb{N}}$ for uma PA constante; será uma PA de ordem 2 se o operador diferença $\left(\Delta^{2} a_{n}\right)_{n \in \mathbb{N}}$ for uma PA constante, e assim por diante, ou, ainda, uma sequência $\left(a_{n}\right)_{n \in \mathbb{N}}$ será uma PA de ordem 2 se $\left(\Delta^{1} a_{n}\right)_{n \in \mathbb{N}}$ for uma PA de primeira ordem; será uma PA de ordem 3 se $\left(\Delta^{2} a_{n}\right)_{n \in \mathbb{N}}$ for uma PA de primeira ordem, e assim por diante.

Exemplo 1. a)A sequência $\left(a_{n}\right)_{n \in \mathbb{N}}=(3,7,13,21,31, \ldots)$, onde $a_{1}=3$ e $a_{n+1}=a_{n}+2 n+2$, para $n \geq 1$, é uma PA de ordem 2 , pois, $\forall n \in \mathbb{N}, \Delta^{1} a_{n}=a_{n+1}-a_{n}=2 n+2, \Delta^{2} a_{n}=\Delta^{1} a_{n+1}-\Delta^{1} a_{n}=2$, e assim, $\left(\Delta^{1} a_{n}\right)_{n \in \mathbb{N}}=(4,6,8,10, \ldots)$ e $\left(\Delta^{2} a_{n}\right)_{n \in \mathbb{N}}=(2,2,2,2, \ldots)$;

b) A sequência $\left(b_{n}\right)_{n \in \mathbb{N}}=(2,8,20,40,70, \ldots)$, onde $b_{1}=2$ e $b_{n+1}=b_{n}+n^{2}+3 n+2$, para $n \geq 1$, é uma PA de ordem 3 , pois, $\forall n \in \mathbb{N}, \Delta^{1} b_{n}=b_{n+1}-b_{n}=n^{2}+3 n+2, \Delta^{2} b_{n}=\Delta^{1} b_{n+1}-\Delta^{1} b_{n}=2 n+4$, $\Delta^{3} b_{n}=\Delta^{2} b_{n+1}-\Delta^{2} b_{n}=2$, e assim, $\left(\Delta^{1} b_{n}\right)_{n \in \mathbb{N}}=(6,12,20,30,42, \ldots),\left(\Delta^{2} b_{n}\right)_{n \in \mathbb{N}}=(6,8,10,12, .$. $\mathrm{e}\left(\Delta^{3} b_{n}\right)_{n \in \mathbb{N}}=(2,2,2,2, \ldots)$;

c) A sequência $\left(c_{n}\right)_{n \in \mathbb{N}}=(1,6,20,50, \ldots)$, onde $c_{1}=1$ e $c_{n+1}=c_{n}+(1 / 6)\left(2 n^{3}+9 n^{2}+13 n+6\right)$, para $n \geq 1$, é uma PA de ordem 4 , pois, $\forall n \in \mathbb{N}, \Delta^{1} c_{n}=c_{n+1}-c_{n}=(1 / 6)\left(2 n^{3}+9 n^{2}+13 n+6\right), \Delta^{2} c_{n}=$ $\Delta^{1} c_{n+1}-\Delta^{1} c_{n}=n^{2}+4 n+4, \Delta^{3} c_{n}=\Delta^{2} c_{n+1}-\Delta^{2} c_{n}=2 n+5, \Delta^{4} c_{n}=\Delta^{3} c_{n+1}-\Delta^{3} c_{n}=2$, e as$\operatorname{sim},\left(\Delta^{1} c_{n}\right)_{n \in \mathbb{N}}=(5,14,30,55,91, \ldots),\left(\Delta^{2} c_{n}\right)_{n \in \mathbb{N}}=(9,16,25,36, \ldots),\left(\Delta^{3} c_{n}\right)_{n \in \mathbb{N}}=(7,9,11,13, \ldots)$ $\mathrm{e}\left(\Delta^{4} c_{n}\right)_{n \in \mathbb{N}}=(2,2,2,2, \ldots)$;

d) A sequência $\left(d_{n}\right)_{n \in \mathbb{N}}=(-1,0,16,97,353, \ldots)$, onde $d_{1}=-1$ e $d_{n+1}=d_{n}+n^{4}$, para $n \geq 1$, é uma PA de ordem 5 , pois, $\forall n \in \mathbb{N}, \Delta^{1} d_{n}=d_{n+1}-d_{n}=n^{4}, \Delta^{2} d_{n}=\Delta^{1} d_{n+1}-\Delta^{1} d_{n}=4 n^{3}+6 n^{2}+4 n+1$, $\Delta^{3} d_{n}=\Delta^{2} d_{n+1}-\Delta^{2} d_{n}=12 n^{2}+24 n+14, \Delta^{4} d_{n}=\Delta^{3} d_{n+1}-\Delta^{3} d_{n}=24 n+36, \Delta^{5} d_{n}=$ 24 , e assim, $\left(\Delta^{1} d_{n}\right)_{n \in \mathbb{N}}=(1,16,81,256, \ldots),\left(\Delta^{2} d_{n}\right)_{n \in \mathbb{N}}=(15,65,175,369, \ldots),\left(\Delta^{3} d_{n}\right)_{n \in \mathbb{N}}=$ $(50,110,194,302, \ldots),\left(\Delta^{4} d_{n}\right)_{n \in \mathbb{N}}=(60,84,108,132, \ldots)$ e $\left(\Delta^{5} d_{n}\right)_{n \in \mathbb{N}}=(24,24,24,24, \ldots)$.

Em seguida, enunciamos uma importante relação entre as PAs de ordem superior e as funções polinomiais, cuja demonstração pode ser encontrada em [4].

Proposição 1. Se $\left(a_{n}\right)_{n \in \mathbb{N}}$ é uma PA de ordem $k$, então seu termo geral $a_{n}$ é um polinômio de grau $k$ na variável $n$. Reciprocamente, se $P(n)$ é um polinômio de grau $k$, então a sequência

$$
\left(a_{n}\right)_{n \in \mathbb{N}}=(P(1), P(2), P(3), \ldots, P(n), \ldots)
$$

é uma PA de ordem $k$.

Com o objetivo de ilustrar essa Proposição, apresentamos o seguinte exercício. 
Exercício 1. a) Supondo que uma sequência $\left(a_{n}\right)_{n \in \mathbb{N}}$ seja uma PA de ordem 2 e que seus três primeiros termos sejam dados por $a_{1}=5, a_{2}=8$ e $a_{3}=13$, obtenha o polinômio de grau 2 que represente o termo geral $a_{n}$.

b) Dado o polinômio $P(n)=n^{3}-3 n^{2}+1$, verifique que a sequência $\left(a_{n}\right)_{n \in \mathbb{N}}=(P(1), P(2), \ldots, P(n), \ldots)$ é uma PA de ordem 3.

\section{Solução:}

a) Como $\left(a_{n}\right)_{n \in \mathbb{N}}$ é uma PA de ordem 2, pela Proposição 1, devemos ter $a_{n}=P(n)=a n^{2}+b n+c$. Assim, $P(1)=5, P(2)=8$ e $P(3)=13$, ou seja,

$$
\left\{\begin{array}{l}
a+b+c=5 \\
4 a+2 b+c=8 \\
9 a+3 b+c=13
\end{array}\right.
$$

Verifica-se que a única solução desse sistema linear é $a=1, b=0$ e $c=4$. Portanto, $a_{n}=n^{2}+4$. b) Como $a_{n}=P(n)=n^{3}-3 n^{2}+1$, temos: $\forall n \in \mathbb{N}, \Delta^{1} a_{n}=P(n+1)-P(n)=3 n^{2}-3 n-2, \Delta^{2} a_{n}=$ $\Delta^{1} a_{n+1}-\Delta^{1} a_{n}=6 n$ e $\Delta^{3} a_{n}=\Delta^{2} a_{n+1}-\Delta^{2} a_{n}=6$. Logo, $\left(a_{n}\right)_{n \in \mathbb{N}}=(-1,-3,1,17,51, \cdots)$, $\left(\Delta^{1} a_{n}\right)_{n \in \mathbb{N}}=(-2,4,16,34, \cdots), \quad\left(\Delta^{2} a_{n}\right)_{n \in \mathbb{N}}=(6,12,18,24, \cdots)$ e $\left(\Delta^{3} a_{n}\right)_{n \in \mathbb{N}}=(6,6,6, \cdots)$. Portanto, $\left(a_{n}\right)_{n \in \mathbb{N}}$ é uma PA de ordem 3 .

\subsection{Termo geral de PAs de ordem superior}

A fórmula para o termo geral de PAs de ordem superior, a qual envolve coeficientes binomiais, será útil para a resolução de diversos problemas. A seguir, enunciamos o termo geral das PAs de ordens 1, 2, 3, 4 e o termo geral de uma PA de ordem $k$. As demonstrações podem ser conferidas em [5, Seção 3.5].

Se $\left(a_{n}\right)_{n \in \mathbb{N}}$ é uma PA de primeira ordem de razão $r$, então $a_{n}=a_{1}+(n-1) r$, ou seja,

$$
a_{n}=\left(\begin{array}{c}
n-1 \\
0
\end{array}\right) a_{1}+\left(\begin{array}{c}
n-1 \\
1
\end{array}\right) r
$$

Se $\left(a_{n}\right)_{n \in \mathbb{N}}$ é uma PA de segunda ordem e $\left(\Delta^{1} a_{n}\right)_{n \in \mathbb{N}}=\left(b_{1}, \ldots, b_{n}, \ldots\right)$ e $\left(\Delta^{2} b_{n}\right)_{n \in \mathbb{N}}=(r, \ldots, r, \ldots)$ são operadores diferenças associados à $\left(a_{n}\right)_{n \in \mathbb{N}}$, então

$$
a_{n}=\left(\begin{array}{c}
n-1 \\
0
\end{array}\right) a_{1}+\left(\begin{array}{c}
n-1 \\
1
\end{array}\right) b_{1}+\left(\begin{array}{c}
n-1 \\
2
\end{array}\right) r
$$

Se $\left(a_{n}\right)_{n \in \mathbb{N}}$ é uma PA de terceira ordem e $\left(\Delta^{1} a_{n}\right)_{n \in \mathbb{N}}=\left(b_{1}, \ldots, b_{n}, \ldots\right),\left(\Delta^{2} a_{n}\right)_{n \in \mathbb{N}}=\left(c_{1}, \ldots, c_{n}, \ldots\right)$ e $\left(\Delta^{3} a_{n}\right)=(r, \ldots, r, \ldots)$ são operadores diferenças associados à $\left(a_{n}\right)_{n \in \mathbb{N}}$, então

$$
a_{n}=\left(\begin{array}{c}
n-1 \\
0
\end{array}\right) a_{1}+\left(\begin{array}{c}
n-1 \\
1
\end{array}\right) b_{1}+\left(\begin{array}{c}
n-1 \\
2
\end{array}\right) c_{1}+\left(\begin{array}{c}
n-1 \\
3
\end{array}\right) r
$$

Se $\left(a_{n}\right)_{n \in \mathbb{N}}$ é uma PA de quarta ordem e $\left(\Delta^{1} a_{n}\right)_{n \in \mathbb{N}}=\left(b_{1}, \ldots, b_{n}, \ldots\right),\left(\Delta^{2} a_{n}\right)_{n \in \mathbb{N}}=\left(c_{1}, \ldots, c_{n}, \ldots\right)$, $\left(\Delta^{3} a_{n}\right)_{n \in \mathbb{N}}=\left(d_{1}, \ldots, d_{n}, \ldots\right)$ e $\left(\Delta^{4} a_{n}\right)_{n \in \mathbb{N}}=(r, \ldots, r, \ldots)$ são operadores diferenças associados à 
$\left(a_{n}\right)_{n \in \mathbb{N}}$, então

$$
a_{n}=\left(\begin{array}{c}
n-1 \\
0
\end{array}\right) a_{1}+\left(\begin{array}{c}
n-1 \\
1
\end{array}\right) b_{1}+\left(\begin{array}{c}
n-1 \\
2
\end{array}\right) c_{1}+\left(\begin{array}{c}
n-1 \\
3
\end{array}\right) d_{1}+\left(\begin{array}{c}
n-1 \\
4
\end{array}\right) r
$$

De forma geral, temos:

Se $\left(a_{n}\right)_{n \in \mathbb{N}}$ é uma PA de ordem $k$ e $\left(\Delta^{1} a_{n}\right)_{n \in \mathbb{N}}=\left(\Delta^{1} a_{1}, \ldots, \Delta^{1} a_{n}, \ldots\right),\left(\Delta^{2} a_{n}\right)_{n \in \mathbb{N}}=\left(\Delta^{2} a_{1}, \ldots\right.$ $\left.\Delta^{2} a_{n}, \ldots\right),\left(\Delta^{k-1} a_{n}\right)_{n \in \mathbb{N}}=\left(\Delta^{k-1} a_{1}, \ldots, \Delta^{k-1} a_{n}, \ldots\right)$ e $\left(\Delta^{k} a_{n}\right)_{n \in \mathbb{N}}=(r, \ldots, r, \ldots)$ são operadores diferenças associados à $\left(a_{n}\right)_{n \in \mathbb{N}}$, então

$$
a_{n}=\left(\begin{array}{c}
n-1 \\
0
\end{array}\right) a_{1}+\left(\begin{array}{c}
n-1 \\
1
\end{array}\right) \Delta^{1} a_{1}+\left(\begin{array}{c}
n-1 \\
2
\end{array}\right) \Delta^{2} a_{1}+\ldots+\left(\begin{array}{c}
n-1 \\
k-1
\end{array}\right) \Delta^{k-1} a_{1}+\left(\begin{array}{c}
n-1 \\
k
\end{array}\right) r .
$$

Exemplo 2. No Exemplo 1, as sequências $\left(a_{n}\right)_{n \in \mathbb{N}}=(3,7,13,21,31, \ldots),\left(b_{n}\right)_{n \in \mathbb{N}}=(2,8,20,40, \ldots)$, $\left(c_{n}\right)_{n \in \mathbb{N}}=(1,6,20,50,105, \ldots)$ e $\left(d_{n}\right)_{n \in \mathbb{N}}=(-1,0,16,97,353, \ldots)$ são apresentadas na forma recursiva, as quais são PAs de ordens 2, 3, 4 e 5, respectivamente. Portanto, por (2), (3), (4) e (5), temos:

$$
\begin{aligned}
& a_{n}=\left(\begin{array}{c}
n-1 \\
0
\end{array}\right) 3+\left(\begin{array}{c}
n-1 \\
1
\end{array}\right) 4+\left(\begin{array}{c}
n-1 \\
2
\end{array}\right) 2 \\
& =\frac{(n-1) !}{0 !(n-1) !} 3+\frac{(n-1) !}{1 !(n-2) !} 4+\frac{(n-1) !}{2 !(n-3) !} 2 \\
& =n^{2}+n+1 \text {, } \\
& b_{n}=\left(\begin{array}{c}
n-1 \\
0
\end{array}\right) 2+\left(\begin{array}{c}
n-1 \\
1
\end{array}\right) 6+\left(\begin{array}{c}
n-1 \\
2
\end{array}\right) 6+\left(\begin{array}{c}
n-1 \\
3
\end{array}\right) 2 \\
& =\frac{(n-1) !}{0 !(n-1) !} 2+\frac{(n-1) !}{1 !(n-2) !} 6+\frac{(n-1) !}{2 !(n-3) !} 6+\frac{(n-1) !}{3 !(n-4) !} 2 \\
& =\frac{1}{3}\left(n^{3}+3 n^{2}+2 n\right), \\
& c_{n}=\left(\begin{array}{c}
n-1 \\
0
\end{array}\right)+\left(\begin{array}{c}
n-1 \\
1
\end{array}\right) 5+\left(\begin{array}{c}
n-1 \\
2
\end{array}\right) 9+\left(\begin{array}{c}
n-1 \\
3
\end{array}\right) 7+\left(\begin{array}{c}
n-1 \\
4
\end{array}\right) 2 \\
& =\frac{(n-1) !}{0 !(n-1) !} 1+\frac{(n-1) !}{1 !(n-2) !} 5+\frac{(n-1) !}{2 !(n-3) !} 9+\frac{(n-1) !}{3 !(n-4) !} 7+\frac{(n-1) !}{4 !(n-5) !} 2 \\
& =\frac{1}{12}\left(n^{4}+4 n^{3}+5 n^{2}+2 n\right),
\end{aligned}
$$




$$
\begin{aligned}
d_{n} & =\left(\begin{array}{c}
n-1 \\
0
\end{array}\right)(-1)+\left(\begin{array}{c}
n-1 \\
1
\end{array}\right) 1+\left(\begin{array}{c}
n-1 \\
2
\end{array}\right) 15+\left(\begin{array}{c}
n-1 \\
3
\end{array}\right) 50+\left(\begin{array}{c}
n-1 \\
4
\end{array}\right) 60+\left(\begin{array}{c}
n-1 \\
5
\end{array}\right) 24 \\
& =\frac{(n-1) !}{0 !(n-1) !}(-1)+\frac{(n-1) !}{1 !(n-2) !}+\frac{(n-1) !}{2 !(n-3) !} 15+\frac{(n-1) !}{3 !(n-4) !} 50+\frac{(n-1) !}{4 !(n-5) !} 60+\frac{(n-1) !}{5 !(n-6) !} 24 \\
& =\frac{1}{30}\left(6 n^{5}-15 n^{4}+10 n^{3}-n-30\right) .
\end{aligned}
$$

Observação 1. Dado que o termo geral $\left(a_{n}\right)_{n \in \mathbb{N}}$ de uma PA de ordem $k$ é um polinômio de grau $k$ na variável $n$ (Proposição 1), podemos calcular o termo geral dessas PAs por meio da resolução de sistemas de equações lineares. No entanto, tal procedimento é muito mais árduo que o ora exposto. Por exemplo, a sequência $\left(c_{n}\right)_{n \in \mathbb{N}}=(1,6,20,50,105, \ldots)$ do Exemplo 1 é uma PA de ordem 4. Portanto, seu termo geral é da forma $c(n)=a n^{4}+b n^{3}+c n^{2}+d n+e$. Assim, devemos ter $c(1)=1, c(2)=6, c(3)=20, c(4)=50, c(5)=105$, ou seja,

$$
\left\{\begin{array}{l}
a+b+c+d+e=1 \\
16 a+8 b+4 c+2 d+e=6 \\
81 a+27 b+9 c+3 d+e=20 \\
256 a+64 b+16 c+4 d+e=50 \\
625 a+125 b+25 c+5 d+e=105 .
\end{array}\right.
$$

Existem diversos métodos (por exemplo, o método de eliminação de Gauss) para resolver esse sistema linear, que tem como solução $a=1 / 12, b=1 / 3, c=5 / 12, d=1 / 6$ e $e=0$ e, portanto,

$$
c_{n}=(1 / 12)\left(n^{4}+4 n^{3}+5 n^{2}+2 n\right) .
$$

Para PAs de ordem elevada, a resolução só é viável computacionalmente. Por exemplo, para uma PA de ordem 9, deve-se resolver um sistema de $10 \times 10$ equações lineares.

Exercício 2. Seja $\left(a_{n}\right)_{n \in \mathbb{N}}=(-9,-2,17,54, \cdots)$, onde $a_{1}=-9$ e $a_{n+1}=a_{n}+3 n^{2}+3 n+1$, para $n \geq 1$, e considere a sequência $\left(S_{n}\right)_{n \in \mathbb{N}}$, onde $S_{n}=a_{1}+a_{2}+\ldots+a_{n}$, a sequência das somas parciais de $\left(a_{n}\right)_{n \in \mathbb{N}}$.

a) Verifique que $\left(a_{n}\right)_{n \in \mathbb{N}}$ é uma PA de ordem 3 .

b) Verifique que $\left(S_{n}\right)_{n \in \mathbb{N}}$ é uma PA de ordem 4 .

c) Calcule a soma dos 15 primeiros termos de $\left(a_{n}\right)_{n \in \mathbb{N}}$.

\section{Solução:}

a) Pela Def. 2, é suficiente observarmos o seguinte: $\forall n \in \mathbb{N}, \Delta^{1} a_{n}=a_{n+1}-a_{n}=3 n^{2}+$ $3 n+1, \Delta^{2} a_{n}=\Delta^{1} a_{n+1}-\Delta^{1} a_{n}=6 n+6, \Delta^{3} a_{n}=\Delta^{2} a_{n+1}-\Delta^{2} a_{n} \equiv 6$, isto é, $\left(\Delta^{1} a_{n}\right)_{n \in \mathbb{N}}=$ $(7,19,37,61,91, \cdots),\left(\Delta^{2} a_{n}\right)_{n \in \mathbb{N}}=(12,18,24,30, \cdots)$ e $\left(\Delta^{3} a_{n}\right)_{n \in \mathbb{N}}=(6,6,6, \cdots)$.

b) Observemos que $\forall n \in \mathbb{N}, \Delta^{1} S_{n}=S_{n+1}-S_{n}=a_{n+1}=a_{n}+3 n^{2}+3 n+1, \Delta^{2} S_{n}=$ $\Delta^{1} S_{n+1}-\Delta^{1} S_{n}=a_{n+1}+3(n+1)^{2}+3(n+1)+1-\left(a_{n}+3 n^{2}+3 n+1\right)=a_{n+1}-a_{n}+6 n+6=3 n^{2}+9 n+7$, $\Delta^{3} S_{n}=\Delta^{2} S_{n+1}-\Delta^{2} S_{n}=6 n+12, \Delta^{4} S_{n}=\Delta^{3} S_{n+1}-\Delta^{3} S_{n} \equiv 6$, isto é, $\left(\Delta^{1} S_{n}\right)_{n \in \mathbb{N}}=$ $(-2,17,54,115,206, \cdots),\left(\Delta^{2} S_{n}\right)_{n \in \mathbb{N}}=(19,37,61,91,127, \cdots),\left(\Delta^{3} S_{n}\right)_{n \in \mathbb{N}}=(18,24,30,36, \cdots)$ e $\left(\Delta^{4} S_{n}\right)_{n \in \mathbb{N}}=(6,6,6,6, \cdots)$. Portanto, $\left(S_{n}\right)_{n \in \mathbb{N}}$ é uma PA de ordem 4 . 
c) Desejamos calcular o $15^{\circ}$ termo da sequência $\left(S_{n}\right)_{n \in \mathbb{N}}$, isto é, o $S_{15}$. Pelo item b), $\left(S_{n}\right)_{n \in \mathbb{N}}$ é uma PA de ordem 4. Assim, por (4),

$$
\begin{aligned}
S_{n} & =\left(\begin{array}{c}
n-1 \\
0
\end{array}\right)(-9)+\left(\begin{array}{c}
n-1 \\
1
\end{array}\right)(-2)+\left(\begin{array}{c}
n-1 \\
2
\end{array}\right) 19+\left(\begin{array}{c}
n-1 \\
3
\end{array}\right) 18+\left(\begin{array}{c}
n-1 \\
4
\end{array}\right) 6 \\
& =\frac{1}{4}\left(n^{4}+2 n^{3}+n^{2}-40 n\right) .
\end{aligned}
$$

Portanto, $S_{15}=\frac{1}{4}\left(15^{4}+2.15^{3}+15^{2}-40.15\right)=14250$.

O exercício anterior nos fornece um exemplo de uma PA de ordem 3, cuja sequência das somas parciais associada é uma PA de ordem 4, o que é verdade em geral, conforme a Proposição a seguir.

Proposição 2. Se $\left(a_{n}\right)_{n \in \mathbb{N}}$ é uma PA de ordem $k$, então a sequência das somas parciais de $\left(a_{n}\right)_{n \in \mathbb{N}}$, denotada por $\left(S_{n}\right)_{n \in \mathbb{N}}$, onde $S_{n}=a_{1}+a_{2}+\ldots+a_{n}$, é uma PA de ordem $k+1$.

Prova: Seja $\left(a_{n}\right)_{n \in \mathbb{N}}=\left(a_{1}, a_{2}, \cdots, a_{n}, \cdots\right)$ uma PA de ordem $k$ e considere

$$
\left(S_{n}\right)_{n \in \mathbb{N}}=\left(a_{1}, a_{1}+a_{2}, a_{1}+a_{2}+a_{3}, \cdots\right) .
$$

Observe que $\left(\Delta^{1} S_{n}\right)_{n \in \mathbb{N}}=\left(a_{2}, a_{3}, a_{4}, \ldots\right)$. Assim, $\left(\Delta^{1} S_{n}\right)_{n \in \mathbb{N}}$ é uma PA de ordem $k$ e, portanto, $\left(S_{n}\right)_{n \in \mathbb{N}}$ é uma PA de ordem $k+1$.

\section{Aplicações}

Nesta seção, apresentaremos alguns problemas que podem ser modelados por PAs de ordem superior. Observamos que, de posse da fórmula do termo geral que envolve coeficientes binomiais, os cálculos que conduzem às soluções desses problemas se tornam bem simples.

Problema 1 (Números Poligonais). Os Números Poligonais são casos particulares de Números Figurados. Denominam-se Números Figurados aqueles que podem ser representados por uma construção geométrica de pontos equidistantes. Caso tal arranjo seja um polígono regular, esses números são chamados de Números Poligonais. A Figura 2 apresenta as sequência dos números triangulares, quadrados, pentagonais e hexagonais. 


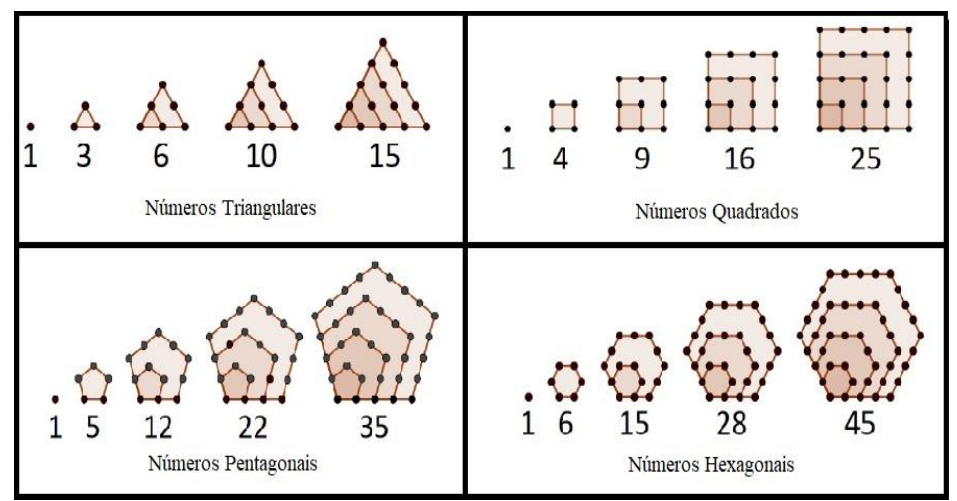

Figura 2: Amostra de números poligonais FONTE: [5].

Verifique que as sequências formadas por esses números poligonais são PAs de segunda ordem e obtenha os seus respectivos termos gerais.

Solução: Analisando a Figura 2, observe que as sequências formadas pelos números triangulares, quadrados, pentagonais e hexagonais são dadas por $\left(T_{n}\right)_{n \in \mathbb{N}}=(1,3,6,10,15, \ldots)$, onde $T_{n}=1+$ $2+\ldots+n ;\left(Q_{n}\right)_{n \in \mathbb{N}}=(1,4,9,16,25, \ldots)$, onde $Q_{n}=1+3+5+\ldots+(2 n-1) ;\left(P_{n}\right)_{n \in \mathbb{N}}=$ $(1,5,12,22,35, \ldots)$, onde $P_{n}=1+4+7+\ldots+(3 n-2)$, e $\left(H_{n}\right)_{n \in \mathbb{N}}=(1,6,15,28,45, \ldots)$, onde $H_{n}=1+5+9+\ldots+(4 n-3)$, respectivamente. Então, $\forall n \in \mathbb{N}, \Delta^{1} T_{n}=n+1, \Delta^{2} T_{n}=$ $1, \Delta^{1} Q_{n}=2 n+1, \Delta^{2} Q_{n}=2, \Delta^{1} P_{n}=3 n+1, \Delta^{2} P_{n}=3, \Delta^{1} H_{n}=4 n+1$ e $\Delta^{2} H_{n}=4$. Assim, $\left(\Delta^{1} T_{n}\right)_{n \in \mathbb{N}}=(2,3,4,5, \ldots),\left(\Delta^{2} T_{n}\right)_{n \in \mathbb{N}}=(1,1,1,1, \ldots),\left(\Delta^{1} Q_{n}\right)_{n \in \mathbb{N}}=(3,5,7,9,11, \ldots)$, $\left(\Delta^{2} Q_{n}\right)_{n \in \mathbb{N}}=(2,2,2,2, \ldots),\left(\Delta^{1} P_{n}\right)_{n \in \mathbb{N}}=(4,7,10,13, \ldots),\left(\Delta^{2} P_{n}\right)_{n \in \mathbb{N}}=(3,3,3, \ldots),\left(\Delta^{1} H_{n}\right)_{n \in \mathbb{N}}=$ $(5,9,13,17, \ldots)$ e $\left(\Delta^{2} H_{n}\right)_{n \in \mathbb{N}}=(4,4,4,4 \ldots)$. Logo, as sequências $\left(T_{n}\right)_{n \in \mathbb{N}},\left(Q_{n}\right)_{n \in \mathbb{N}},\left(P_{n}\right)_{n \in \mathbb{N}} \mathrm{e}$ $\left(H_{n}\right)_{n \in \mathbb{N}}$ são PAs de segunda ordem. Portanto, por (2),

$$
\begin{aligned}
T_{n} & =\left(\begin{array}{c}
n-1 \\
0
\end{array}\right) 1+\left(\begin{array}{c}
n-1 \\
1
\end{array}\right) 2+\left(\begin{array}{c}
n-1 \\
2
\end{array}\right) 1 \\
& =\frac{1}{2}\left(n^{2}+n\right) \\
Q_{n} & =\left(\begin{array}{c}
n-1 \\
0
\end{array}\right) 1+\left(\begin{array}{c}
n-1 \\
1
\end{array}\right) 3+\left(\begin{array}{c}
n-1 \\
2
\end{array}\right) 2 \\
& =n^{2},
\end{aligned}
$$




$$
\begin{aligned}
P_{n} & =\left(\begin{array}{c}
n-1 \\
0
\end{array}\right) 1+\left(\begin{array}{c}
n-1 \\
1
\end{array}\right) 4+\left(\begin{array}{c}
n-1 \\
2
\end{array}\right) 3 \\
& =\frac{3 n^{2}-n}{2} \\
H_{n} & =\left(\begin{array}{c}
n-1 \\
0
\end{array}\right) 1+\left(\begin{array}{c}
n-1 \\
1
\end{array}\right) 5+\left(\begin{array}{c}
n-1 \\
2
\end{array}\right) 4 \\
& =2 n^{2}-n .
\end{aligned}
$$

Problema 2 (Castelo de cartas). A Figura 3 mostra castelos de cartas de 1, 2 e 3 andares. De quantos baralhos de 52 cartas precisamos, no mínimo, para construir um castelo de 10 andares?
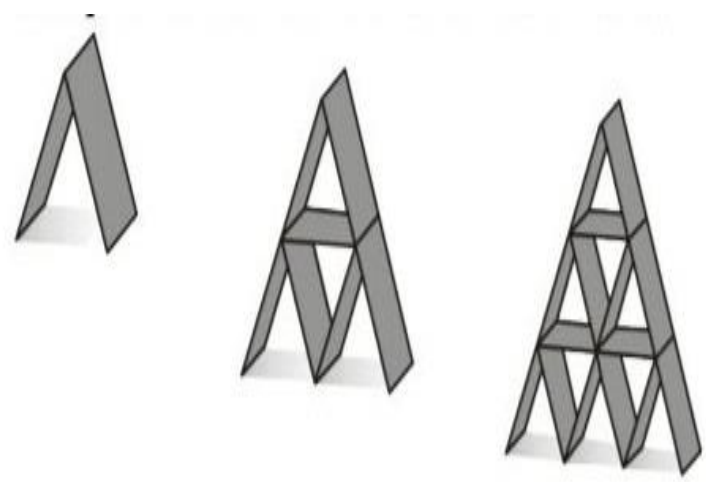

Figura 3: Castelo de Cartas, FONTE: OBMEP-2009.

Solução: Seguindo a lógica da Figura 3, deduz que o $n$-ésimo termo da sequência $\left(a_{n}\right)_{n \in \mathbb{N}}$, onde $a_{1}=2$ e $\forall n \in \mathbb{N}, a_{n+1}=a_{n}+3 n+2$, isto é, $\left(a_{n}\right)_{n \in \mathbb{N}}=(2,7,15,26,40, \ldots)$, representa a quantidade mínima de cartas para construir um castelo de $n$ andares. Observe que, $\forall n \in \mathbb{N}, \Delta^{1} a_{n}=3 n+2$ e $\Delta^{2} a_{n} \equiv 3$, isto é, $\left(\Delta^{1} a_{n}\right)_{n \in \mathbb{N}}=(5,8,11,14, \ldots)$ e $\left(\Delta^{2} a_{n}\right)_{n \in \mathbb{N}}=(3,3,3,3, \ldots)$. Logo, $\left(a_{n}\right)_{n \in \mathbb{N}}$ é uma PA de segunda ordem. Assim, por (2),

$$
\begin{aligned}
a_{n} & =\left(\begin{array}{c}
n-1 \\
0
\end{array}\right) 2+\left(\begin{array}{c}
n-1 \\
1
\end{array}\right) 5+\left(\begin{array}{c}
n-1 \\
2
\end{array}\right) 3 \\
& =\frac{1}{2}\left(3 n^{2}+n\right) .
\end{aligned}
$$

Portanto, $a_{10}=(1 / 2)\left(3 \times 10^{2}+10\right)=155$ representa o número mínimo de cartas para construir um castelo de 10 andares. Como um baralho tem 52 cartas, serão necessários, no mínimo, 3 baralhos para construir um castelo de 10 andares. 
Problema 3 (Lançamento oblíquo). Um operador de canhão está fazendo testes para medir a relação entre a quantidade de pólvora usada na explosão e a distância atingida pela bala quando o canhão está com inclinação de 45 graus. Ele já sabe que, para cada meio quilo de pólvora, há uma variação

$10 \mathrm{~m} / \mathrm{s}$ na velocidade de lançamento. Quanto de pólvora deve ser utilizada no canhão para que o projétil ultrapasse 300 metros? A Figura 4 ilustra esse problema e a Tabela 1 mostra algumas relações que foram calculadas pelo operador.

Nota: após alguns testes, o operador de canhão percebeu que a sequência das distâncias atingidas $\left(D_{n}\right)_{n \in \mathbb{N}}$ obedece ao seguinte padrão: $D_{1}=10.2 \mathrm{e}, \forall n \in \mathbb{N}, D_{n+1}=D_{n}+20.41 n+10.2$.

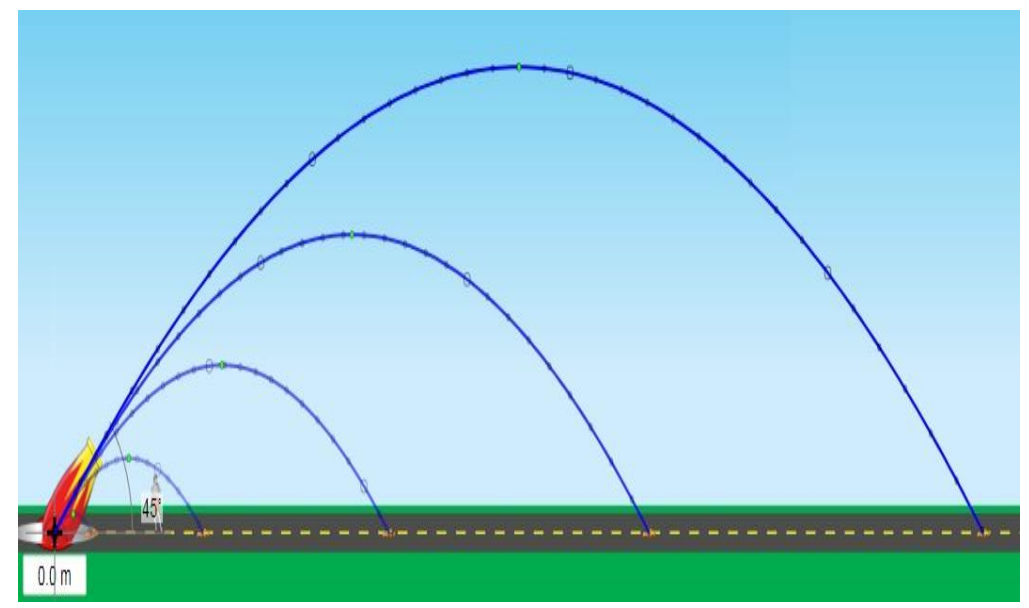

Figura 4: Lançamento Oblíquo, FONTE:[5].

\begin{tabular}{|c|c|c|}
\hline Pólvora $(\mathrm{kg})$ & Velocidade $(\mathrm{m} / \mathrm{s})$ & Distância $(\mathrm{m})$ \\
\hline 0.5 & 10 & 10.2 \\
\hline 1 & 20 & 40.81 \\
\hline 1.5 & 30 & 91.83 \\
\hline 2 & 40 & 163.26 \\
\hline
\end{tabular}

Tabela 1: Dados complementares do Problema 3.

Solução: Seja $\left(D_{n}\right)_{n \in \mathbb{N}}=(10.2,40.81,91.83,163.26, \ldots)$, onde $D_{1}=10.2$ e, $\forall n \in \mathbb{N}, D_{n+1}=$ $D_{n}+20.41 n+10.2$, a sequência das distâncias atingidas. Assim, $\forall n \in \mathbb{N}, \Delta^{1} D_{n}=20.41 n+$ 10.2 e $\Delta^{2} D_{n} \equiv 20.41$, isto é, $\left(\Delta^{1} D_{n}\right)_{n \in \mathbb{N}}=(30.61,51.02,71.43,91.84, \ldots)$ e $\left(\Delta^{2} D_{n}\right)_{n \in \mathbb{N}}=$ 
$(20.41,20.41, \ldots)$. Desse modo, $\left(D_{n}\right)_{n \in \mathbb{N}}$ é uma PA de segunda ordem e, então, por (2),

$$
\begin{aligned}
D_{n} & =\left(\begin{array}{c}
n-1 \\
0
\end{array}\right) 10.2+\left(\begin{array}{c}
n-1 \\
1
\end{array}\right) 30.61+\left(\begin{array}{c}
n-1 \\
2
\end{array}\right) 20.41 \\
& =10.205 n^{2}-0.005 n .
\end{aligned}
$$

Assim, para que o projétil ultrapasse 300 metros, devemos ter $10.205 n^{2}-0.005 n>300$, o que nos leva a $n>5.42$. Pela Tabela $1, v=10 n$. Logo, a velocidade requerida para ultrapassar 300 metros deverá ser maior que $54.2 \mathrm{~m} / \mathrm{s}$, o que equivale a dizer, por proporção, que serão necessários mais que $2.71 \mathrm{~kg}$ de pólvora para que o projétil ultrapasse 300 metros.

Problema 4 (Queda livre). Um objeto foi solto de uma altura $h$, em queda livre. A partir do $3^{\underline{o}}$ segundo de queda, um sistema começou a monitorar as alturas desse objeto em relação ao seu tempo de queda. Alguns dos dados monitorados estão disponíveis na Tabela 2 a seguir.

\begin{tabular}{|c|c|}
\hline \multicolumn{2}{|c|}{ Objeto em Queda Livre } \\
\hline Tempo (s) & Altura (m) \\
\hline 3 & 955 \\
\hline 4 & 920 \\
\hline 5 & 875 \\
\hline 6 & 820 \\
\hline 7 & 755 \\
\hline
\end{tabular}

Tabela 2: Altura em função do tempo de queda.

a) De que altura o objeto foi solto?

b) Quanto tempo o objeto gastará para chegar ao solo?

Solução: Como os tempos fornecidos são consecutivos, podemos interpretar as respectivas alturas como termos de uma sequência. Assim, seja $\left(h_{n}\right)_{n \in \mathbb{N}}=(\ldots, 955,920,875,820,755, \ldots)$, onde $h_{1}=$ 955 e, $\forall n \in \mathbb{N}, h_{n+1}=h_{n}-10 n-25$, a sequência das alturas. Logo, $\forall n \in \mathbb{N}, \Delta^{1} h_{n}=h_{n+1}-h_{n}=$ $-10 n-25$ e $\Delta^{2} h_{n}=\Delta^{1} h_{n+1}-\Delta^{1} h_{n} \equiv-10$, isto é,

$$
\left(\Delta^{1} h_{n}\right)_{n \in \mathbb{N}}=(\ldots,-35,-45,-55,-65, \ldots) \text { e }\left(\Delta^{2} h_{n}\right)_{n \in \mathbb{N}}=(\ldots,-10,-10,-10, \ldots) .
$$

Logo, as alturas formam uma PA de segunda ordem. Portanto, por (2), o termo geral de $\left(h_{n}\right)_{n \in \mathbb{N}}$ é dado por

$$
\begin{aligned}
h_{n} & =\left(\begin{array}{c}
n-1 \\
0
\end{array}\right) 955+\left(\begin{array}{c}
n-1 \\
1
\end{array}\right)(-35)+\left(\begin{array}{c}
n-1 \\
2
\end{array}\right)(-10) \\
& =-5 n^{2}-20 n+980 .
\end{aligned}
$$

Uma vez calculado o termo geral da PA, vamos apresentar as respostas às perguntas formuladas. 
a) Nesse caso, desejamos calcular $h_{(-2)}$ (isso é possível, pois consideramos o terceiro termo da sequência como o primeiro). Portanto,

$$
h_{(-2)}=-5 \cdot(-2)^{2}-20 \cdot(-2)+980=1000,
$$

ou seja, o objeto foi solto a uma altura de 1000 metros.

b) Nesse caso, resolvemos a equação $-5 n^{2}-20 n+980=0$, o que resulta em $n \cong 12.14$. Isto é, o objeto gastará, aproximadamente, $12.14+3=15.14$ segundos para chegar ao solo.

Problema 5 (Espiral de naturais). Os números naturais foram dispostos em uma espiral retangular, como mostra a Figura 5 a seguir. Qual será o número que ocupará a vigésima posição na direção indicada?

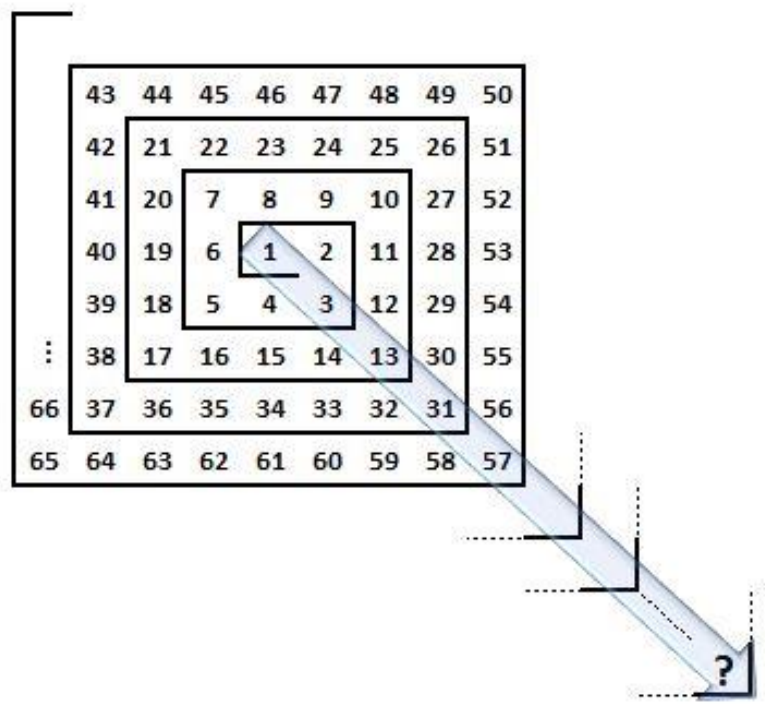

Figura 5: Números Naturais em espiral retangular.

Solução: Seguindo a lógica da Figura 5, observamos que os números indicados formam a sequência $\left(a_{n}\right)_{n \in \mathbb{N}}$, onde $a_{1}=1 \mathrm{e}, \forall n \in \mathbb{N}, a_{n+1}=a_{n}+8 n-6$, isto é, $\left(a_{n}\right)_{n \in \mathbb{N}}=(1,3,13,31,57, \ldots)$. Logo, $\forall n \in \mathbb{N}, \Delta^{1} a_{n}=a_{n+1}-a_{n}=8 n-6$ e $\Delta^{2} a_{n}=\Delta^{1} a_{n+1}-\Delta^{1} a_{n} \equiv 8$ e, assim, $\left(\Delta^{1} a_{n}\right)_{n \in \mathbb{N}}=$ $(2,10,18,26, \ldots) e\left(\Delta^{2} a_{n}\right)_{n \in \mathbb{N}}=(8,8, \ldots)$. Então, $\left(a_{n}\right)_{n \in \mathbb{N}}$ é uma PA de segunda ordem. Daí, por (2), temos:

$$
\begin{aligned}
a_{n} & =\left(\begin{array}{c}
n-1 \\
0
\end{array}\right) 1+\left(\begin{array}{c}
n-1 \\
1
\end{array}\right) 2+\left(\begin{array}{c}
n-1 \\
2
\end{array}\right) 8 \\
& =4 n^{2}-10 n+7 .
\end{aligned}
$$


11

12121

$\begin{array}{llll}1 & 3 & 3 & 1\end{array}$

$\begin{array}{lllll}1 & 4 & 6 & 4 & 1\end{array}$

$\begin{array}{llllll}1 & 5 & 10 & 10 & 5 & 1\end{array}$

$\begin{array}{lllllll}1 & 6 & 15 & 20 & 15 & 6 & 1\end{array}$

$\begin{array}{llllllll}1 & 7 & 21 & 35 & 35 & 21 & 7 & 1\end{array}$

$\begin{array}{lllllllll}1 & 8 & 28 & 56 & 70 & 56 & 28 & 8 & 1\end{array}$

$\begin{array}{llllllllll}1 & 9 & 36 & 84 & 126 & 126 & 84 & 36 & 9 & 1\end{array}$

$\begin{array}{lllllllllll}1 & 10 & 45 & 120 & 210 & 252 & 210 & 120 & 45 & 10 & 1\end{array}$

Figura 6: Triângulo de Pascal, FONTE: [5].

Portanto, $a_{20}=4(20)^{2}-10(20)+7=1407$ é o número procurado.

Na próxima aplicação, vamos usar o Triângulo de Pascal, que é uma estrutura numérica na qual a $n$-ésima linha é composta pelos coeficientes de $(a+b)^{n}$. Desse modo, temos:

$(a+b)^{0}=1$,

$(a+b)^{1}=a+b$,

$(a+b)^{2}=a^{2}+2 a b+b^{2}$,

$(a+b)^{3}=a^{3}+3 a^{2} b+3 a b^{2}+b^{3}$,

$(a+b)^{4}=a^{4}+4 a^{3} b+6 a^{2} b^{2}+4 a b^{3}+b^{4}$,

$(a+b)^{5}=a^{5}+5 a^{4} b+10 a^{3} b^{2}+10 a^{2} b^{3}+5 a b^{4}+b^{5}$.

Prosseguindo analogamente, teremos o triângulo numérico conhecido como o Triângulo de Pascal, cujas primeiras linhas são mostradas na Figura 6 a seguir.

Uma das principais propriedades do Triângulo de Pascal é que a soma de dois números consecutivos de uma linha $l$ resulta no elemento da linha $l+1$ imediatamente abaixo do segundo número somado.

Problema 6 (Triângulo de Pascal). Considere a Figura 6.

a) Mostre que a sequência contida na $5^{a}$ coluna do Triângulo de Pascal é uma PA de quarta ordem.

b) Calcule o termo geral da PA contida na $5^{a}$ coluna do Triângulo de Pascal.

c) Obtenha a expressão que fornece a soma dos $n$ primeiros termos da PA contida na $5^{a}$ coluna.

\section{Solução:}

a) Seja $\left(P_{n}\right)_{n \in \mathbb{N}}=(1,5,15,35,70, \cdots)$, onde $P_{1}=1$ e, $\forall n \in \mathbb{N}, P_{n+1}=P_{n}+(1 / 6)(n+1)(n+2)(n+3)$, 
a sequência contida na $5^{a}$ coluna. Assim, $\forall n \in \mathbb{N}, \Delta^{1} P_{n}=P_{n+1}-P_{n}=(1 / 6)(n+1)(n+2)(n+3)$, $\Delta^{2} P_{n}=\Delta^{1} P_{n+1}-\Delta^{1} P_{n}=(1 / 2)(n+3)(n+2), \Delta^{3} P_{n}=\Delta^{2} P_{n+1}-\Delta^{2} P_{n}=n+3$ e $\Delta^{4} P_{n} \equiv 1$, ou seja, $\left(\Delta^{1} P_{n}\right)_{n \in \mathbb{N}}=(4,10,20,35,56, \ldots),\left(\Delta^{2} P_{n}\right)_{n \in \mathbb{N}}=(6,10,15,21,28, \ldots),\left(\Delta^{3} P_{n}\right)_{n \in \mathbb{N}}=(4,5,6,7,8, \ldots)$ e $\left(\Delta^{4} P_{n}\right)_{n \in \mathbb{N}}=(1,1,1, \ldots)$. Portanto, pela Definição $2,\left(P_{n}\right)_{n \in \mathbb{N}}$ é uma PA de quarta ordem.

b) Com base no item a) e em (4), temos:

$$
\begin{aligned}
P_{n} & =\left(\begin{array}{c}
n-1 \\
0
\end{array}\right) 1+\left(\begin{array}{c}
n-1 \\
1
\end{array}\right) 4+\left(\begin{array}{c}
n-1 \\
2
\end{array}\right) 6+\left(\begin{array}{c}
n-1 \\
3
\end{array}\right) 4+\left(\begin{array}{c}
n-1 \\
4
\end{array}\right) 1 \\
& =\frac{1}{24}\left(n^{4}+6 n^{3}+11 n^{2}+6 n\right) .
\end{aligned}
$$

c) Seja $\left(P_{n}\right)_{n \in \mathbb{N}}$ a sequência contida na $5^{a}$ coluna e $\left(S_{n}\right)_{n \in \mathbb{N}}$ a sequência das somas parciais de $\left(P_{n}\right)_{n \in \mathbb{N}}$. Como $\left(P_{n}\right)_{n \in \mathbb{N}}$ é uma PA de ordem 4, temos que, pela Proposição $2,\left(S_{n}\right)_{n \in \mathbb{N}}$ é uma PA de ordem 5 . Como $S_{1}=1, \Delta^{1} S_{1}=5, \Delta^{2} S_{1}=10, \Delta^{3} S_{1}=10, \Delta^{4} S_{1}=5$ e $\Delta^{5} S_{1}=1$, temos:

$$
\begin{aligned}
S_{n} & =\left(\begin{array}{c}
n-1 \\
0
\end{array}\right) 1+\left(\begin{array}{c}
n-1 \\
1
\end{array}\right) 5+\left(\begin{array}{c}
n-1 \\
2
\end{array}\right) 10+\left(\begin{array}{c}
n-1 \\
3
\end{array}\right) 10+\left(\begin{array}{c}
n-1 \\
4
\end{array}\right) 5+\left(\begin{array}{c}
n-1 \\
5
\end{array}\right) 1 \\
& =\frac{1}{120} n(n+1)(n+2)(n+3)(n+4) .
\end{aligned}
$$

Observação 2. Os elementos de uma coluna $c$ do triângulo de Pascal formam uma PA de ordem $c-1$, em que $c \in\{1,2,3, \ldots\}$.

\section{Considerações finais}

Pelo exposto, entendemos que é necessária uma abordagem apropriada de PAs de ordem superior no Ensino Médio, em especial, para os professores que trabalham com olimpíadas de Matemática, servindo-lhes mesmo de base para a propositura de problemas mais avançados.

\section{Referências}

[1] Contador, P. R. M. 2007. A Matemática na Arte e na Vida. São Paulo: Livraria da Física.

[2] Dlab, V. 2011. Arithmetic progressions of higher order. Teaching Mathematics and Computer Science, v. 4, p. 225-239.

[3] Lima, W. A. F. 2015. Progressões Aritméticas de Ordem Superior: uma proposta de abordagem no Ensino Médio. Dissertação (PROFMAT-UEM), Maringá-PR.

[4] Morgado, A. C. O., Carvalho, P. C. P. 2015. Matemática Discreta. Coleção PROFMAT. Rio de Janeiro: SBM.

[5] Nobre, J. F. F. 2018. Progressões Aritméticas: abordando as ordens superiores. Dissertação (PROFMAT-UFT), Palmas-TO.

[6] Roque, T. 2012. História da Matemática: uma visão crítica, desfazendo mitos e lendas. Rio de Janeiro: Zahar. 
José Nobre

Diretoria Regional de Ensino - Araguatins - TO

$<$ josefilho010@gmail.com>

Rogério Rocha

Universidade Federal do Tocantins - Palmas - TO

<azevedo@uft.edu.br>

Recebido: 02/05/2018 